\title{
INCOMPATIBILIDAD SEXUAL, UN MECANISMO GENÉTICO QUE EVITA LA AUTOFECUNDACIÓN Y CONTRIBUYE A LA DIVERSIDAD VEGETAL
}

\section{SEXUAL INCOMPATIBILITY, A GENETIC MECHANISM THAT PREVENTS SELF- FERTILIZATION AND CONTRIBUTES TO PLANT DIVERSITY}

\author{
Karina Jiménez-Durán y Felipe Cruz-García*
}

Departamento de Bioquímica, Facultad de Química, Universidad Nacional Autónoma de México. Av. Universidad 3000, Col. Copilco. 04510, México D.F. Tel: 55 56225279, Fax: 56225329.

*Autor para correspondencia (fcg@unam.mx.)

\section{RESUMEN}

La proximidad de los órganos sexuales en las flores hermafroditas incrementa considerablemente la probabilidad de la autofecundación. Para evitarla, varias especies desarrollaron el sistema de incompatibilidad sexual (SI), mecanismo presente en varias familias de angiospermas que permite al pistilo rechazar el polen propio y aceptar el de plantas genéticamente diferentes. Esto evita la endogamia y favorece la polinización cruzada. La SI está controlada por un locus muy polimórfico llamado locus $S$ que incluye dos unidades transcripcionales estrechamente ligadas. Una de ellas codifica la determinante masculina y la otra a la determinante femenina. En esta revisión se discuten los avances en el control molecular del SI gametofítico presente en las familias Solanaceae, Rosaceae y Plantaginaceae, en las que la especificidad del rechazo del polen lo ejerce la interacción $S$-específica de la $S$ RNasa (determinante femenina) en el pistilo y la proteína SLF (determinante masculina) en el polen. Además, se revisan los posibles mecanismos bioquímicos que conducen al rechazo del polen propio en sistemas basados en S-RNasa, y se propone un modelo combinado.

Palabras clave: Sistemas de incompatibilidad, rechazo del polen, locus $S$, S-RNasa, SLF.

\section{SUMMARY}

Proximity between sexual organs in hermaphrodite flowers highly increases the likelihood of self-fertilization. To avoid it, several species evolved the sexual incompatibility (SI) system, a mechanism present in several angiosperm families which allows the pistil to reject self-pollen and to accept non-self-pollen, thus encouraging outcrossing to prevent inbreeding. The SI is genetically controlled by male and female determinants, both encoded in the highly polymorphic $S$-locus. In this paper we review and discuss the current advances in the molecular control of the gametophytic SI, present in the Solanaceae, Rosaceae and Plantaginaceae families, in which the specificity of pollen rejection is determined by the $S$ specific interaction between the S-RNase (female determinant) and SLF (S locus F-box protein; male determinant). In addition, we discuss the potential biochemical leading to pollen rejection in SRNase based systems, and we propose a combined model.

Recibido: 22 de Junio del 2010.

Aceptado: 02 de Marzo del 2011.
Index words: Self-incompatibility systems, pollen rejection, $S$ locus, $S$-RNase, SLF.

\section{INTRODUCCIÓN}

Las angiospermas representan el grupo más importante de las plantas vasculares. Su gran diversidad, amplia masculino (estambres) en la misma flor. La cercanía de estas estructuras aumenta la probabilidad de autopolinización y, por ende, de autofecundación. Para regularla, el pistilo juega un papel esencial, ya que además de recibir al polen en la superficie de su estigma, permitir su hidratación, su germinación y la nutrición y conducción de los tubos polínicos al ovario, también sintetiza factores que ayudan a reconocer y discriminar el polen producido por la misma planta de aquel que proviene de individuos genéticamente relacionados y de otras especies (McClure y Frankling-Tong, 2006).

Para promover la polinización cruzada, varias especies hermafroditas desarrollaron adaptaciones morfológicas como la separación espacial del pistilo y los estambres (hercogamia), y fisiológicas, como la maduración diferencial de los órganos reproductivos (dicogamia). $\mathrm{Si}$ bien estas modificaciones evitan en gran medida la autofecundación y promueven la polinización cruzada, aún existe la posibilidad de flujo génico a través del polen con los padres o con otros individuos de la progenie. Para contender con esto, un gran número de especies desarrollaron un mecanismo genético-bioquímico de reconocimiento del polen, conocido como sistema de incompatibilidad sexual (AI), el cual se define como la incapacidad de una planta hermafrodita fértil para 
producir cigotos después de la autopolinización (de Nettancourt, 1977; 2001).

El fuerte control genético que ejercen los sistemas de AI restringe grandemente la introgresión con los padres y las cruzas con otros miembros de la progenie. De esta manera la polinización cruzada aumenta la capacidad de sobrevivencia y reproducción, y contribuye así a la diversidad genética de la siguiente generación. En varias familias la AI está controlada genéticamente por un solo locus multialélico conocido como locus $S$, el cual incluye dos genes estrechamente ligados. Una unidad transcripcional codifica la determinante masculina con expresión específica en el polen, y la otra codifica a la femenina con expresión exclusiva en el pistilo. Esta revisión se enfoca al sistema de incompatibilidad gametofítico, para describir el avance logrado en la identificación de los genes que controlan la vía del rechazo del polen, así como de los mecanismos propuestos que la regulan.

Los datos derivados del estudio del mecanismo que controla el rechazo del polen, podrían ser utilizados en programas de mejoramiento genético de especies de importancia agrícola en las familias Solanaceae, Rosaceae y Poaceae, las cuales incluyen especies incompatibles. Por ejemplo, serviría para caracterizar las vías de flujo de polen entre especies silvestres y cultivables, y así determinar si existen barreras reproductivas que eviten la incorporación de alelos de resistencia a enfermedades o a condiciones ambientales adversas. Este conocimiento permitiría establecer esquemas de cruzas dirigidas que impacten positivamente a la agricultura.

\section{Sistemas genéticos que controlan el rechazo del polen}

Los sistemas de AI se distribuyen ampliamente en las angiospermas y están presentes en 91 de las 271 familias descritas (Dickinson et al., 1992). El número de alelos $\mathrm{S}$ presentes en una población puede ser muy grande y variar de especie a especie; en algunas especies de Brassica existen más de 60 alelos, de 60 a 80 en Papaver y de 150 a 250 en Trifolium (Dickinson et al., 1992). La AI es un ejemplo clásico de un sistema genético que se encuentra bajo selección balanceadora, para mantener los polimorfismos genéticos dentro de una población (Charlesworth et al., 2005; Newbigin y Uyenoyama, 2005).

En especies de la familia Solanaceae, como Solanum lycopersicum (Lycopersicum esculentum L.) y Solanum tuberosum L., el locus $S$ se localiza cerca del centrómero del cromosoma I (Tanksley y Loaiza-Figueroa, 1985), mientras que en Petunia hybrida está en la región pericentromérica del cromosoma III (Entani et al., 1999). La ubicación del locus $S$ cerca del centrómero y la proximidad de las dos determinantes provoca que no haya recombinación entre ambos genes, lo que promueve su herencia como una sola unidad mendeliana llamada haplotipo (Cruz-García y McClure, 2001).

En función del tiempo de expresión de la determinante masculina en el polen, los tipos de SI se clasifican en gametofítico (SIG) y esporofítico (SIS) (Newbigin et al., 1993). En los sistemas SIG los componentes que determinan la incompatibilidad son expresados por el genotipo haploide del grano de polen (gametofito masculino; por eso el nombre de incompatibilidad gametofítica; Figura 1A). En este caso el rechazo del polen ocurre cuando el haplotipo $S$ del polen coincide con uno de los dos haplotipos $S$ presentes en el pistilo diploide de una planta heterocigota receptora, lo que provoca que el crecimiento del tubo polínico (TP) se inhiba generalmente a nivel del estilo. Por ejemplo, una planta diploide $S_{1} S_{2}$ producirá granos de polen genética y fenotípicamente $S_{1}$ o $S_{2}$. Cuando los granos de polen con el haplotipo $S_{1}$ o $S_{2}$ lleguen a un pistilo $S_{1} S_{2}$, germinarán y producirán un TP. Sin embargo, como la cruza es incompatible su crecimiento será inhibido a nivel del estilo. Pero si el haplotipo del polen es $S_{3}$, el polen será aceptado y el TP alcanzará el saco embrionario para llevar a cabo la fecundación, lo que resultará en una progenie con individuos $S_{1} S_{3}$ y $S_{2} S_{3}$ (Cruz-García y McClure, 2001) como se ilustra en la Figura 1A).

En los sistemas SIS la incompatibilidad está determinada por los productos de los alelos $S$ que son expresados y sintetizados por los tejidos diploides de la planta madre, como el tapete, los cuales son incorporados a la cubierta del grano de polen durante su maduración en la antera. En una planta madre heterocigota los dos productos de los alelos $S$ estarán presentes en la cubierta del polen (Figura 1B); en este caso una planta $S_{1} S_{2}$ producirá granos de polen con genotipos $S_{1}$ o $S_{2}$, pero todo el polen producido tendrá el fenotipo $S_{1} S_{2}$. Si los granos de polen cubiertos en su superficie con proteínas $S_{1}$ y $S_{2}$ caen sobre el estigma de una planta $S_{1} S_{2}$ el polen será rechazado, en cuyo caso la respuesta de incompatibilidad se manifiesta con inhibición de la germinación del polen en la superficie del estigma. Pero si el polen lleva el producto de los alelos $S_{3} S_{4}$ en su superficie (pero con un genotipo $S_{3}$ o $S_{4}$ ), el polen germinará y el TP crecerá hasta el óvulo donde ocurrirá la fecundación, para producir una progenie con individuos $S_{1} S_{3}, S_{1} S_{4}, S_{2} S_{3}$ y $S_{2} S_{4}$; en este caso toda la progenie producirá polen con las proteínas $S_{1} S_{3}, S_{1} S_{4}, S_{2} S_{3}$ y $S_{2} S_{4}$ en su superficie, lo que evitará la introgresión con los padres cuyos genotipos son $S_{1} S_{2}$ y $S_{3} S_{4}$, o con sus 
hermanos, ya que siempre habrá coincidencia con uno de los haplotipos $S$. Lo anterior no ocurre en especies SIG, ya que la progenie puede introgresar parcialmente con los padres y con algunos individuos de la progenie (Cruzarcía y McClure, 2001).

\section{Sistemas de autoincompatibilidad}

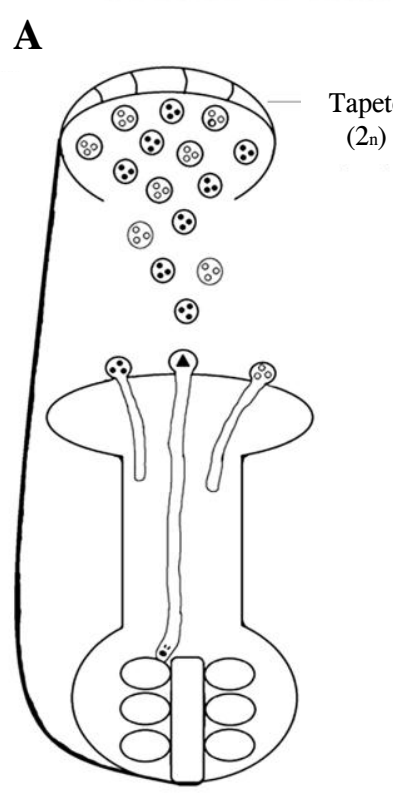

Pistilo $S_{1} S_{2}$

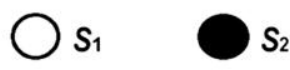

Progenie

$S_{1} S_{3}$

$\mathrm{S}_{2} \mathrm{~S}_{3}$
B

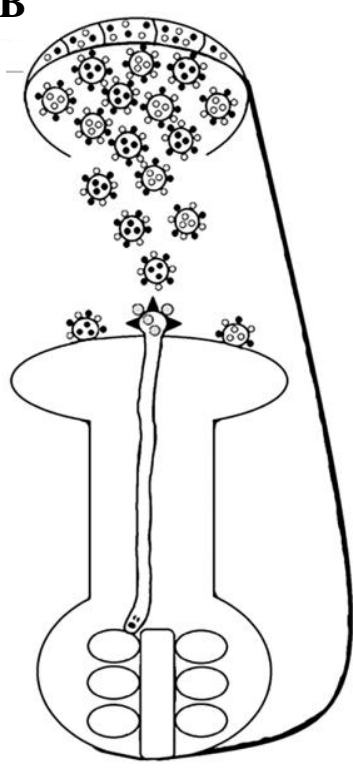

Pistilo $S_{I} S_{2}$

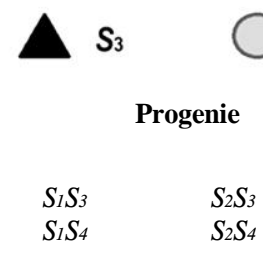

Figura 1. Control genético en los sistemas de incompatibilidad homomórficos. A) Control gametofítico. Los granos de polen contienen en su citoplasma el producto del alelo $S_{1}$ o $S_{2}$. Al llegar al estigma del pistilo $S_{1} S_{2}$, germinan y crecen a través del estigma, pero su crecimiento es inhibido en la parte superior del estilo. Sin embargo, un grano de polen con el alelo $S_{3}$ es capaz de germinar y crecer a través del tejido de transmisión del estilo hasta alcanzar el ovario y fecundar los óvulos, lo que producirá una progenie $S_{1} S_{3}$ y $S_{2} S_{3}$. B) Control esporofítico. El producto del alelo $S_{1}$ y $S_{2}$ se expresa en el tejido diploide del tapete, que al degenerar vierte su contenido al interior del lóculo de la antera y cubre la pared de los granos de polen. De esta forma cada grano de polen presenta los productos de los dos alelos $S$ en su cubierta. Los granos de polen al llegar al estigma de un pistilo $S_{1} S_{2}$ son reconocidos como propios y su germinación se inhibe. $\mathrm{Si}$, por el contrario, llega polen fenotípicamente $S_{3} S_{4}$, estos granos de polen germinan y crecen por el tejido de transmisión del estilo hasta alcanzar los óvulos y fecundarlos, para producir una progenie $S_{1} S_{3}, S_{1} S_{4}, S_{2} S_{3}$ y $S_{2} S_{4}$.

\section{Sistemas de autoincompatibilidad gametofíticos dependientes de S-RNasa}

El sistema AIG es de los más representados en las angiospermas y se ha estudiado fundamentalmente en las familias Solanaceae (Nicotiana, Solanum, Petunia), Plantaginaceae (Antirrhinum) y Rosaceae (Prunus, Pyrus y Malus) (de Nettancourt, 2001; Yamame y Tao, 2009).

S-RNasa es la determinante femenina. El producto génico de la determinante femenina en las familias Rosaceae, Plantaginaceae y Solanaceae, es una proteína con actividad de ribonucleasa conocida como S-RNasa (McClure et al., 1989; Kao y McCubbin, 1996). Las SRNasas son glicoproteínas de aproximadamente $30 \mathrm{kD}$ que se expresan en estigma, y ovario, y se localizan en la matriz extracelular del tejido de transmisión del estilo (Anderson et al., 1986; Cornish et al., 1988; McClure et al., 1989; Sassa et al., 1996; Xue et al., 1996).

Experimentos de ganancia o pérdida de función con plantas transgénicas en Solanum, Petunia y Nicotiana, demuestran que la expresión de diferentes S-RNasas, causa cambios en el fenotipo de polinización, para pasar de compatible a incompatible o viceversa. Por ejemplo, la transformación de los híbridos de Nicotiana langsdorffii x $N$. alata $S_{105} S_{105}$ que expresan los transgenes que codifican a la $S_{2}$-RNasa o a la $S_{110}$ RNasa, rechazan el polen $S_{\mathrm{A} 2}$ o $S_{\mathrm{C} 10}$, fenotipo que las plantas sin transformar no presentan (Murfett et al., 1994; 1996). En las especies Prunus, Pyrus y Malus de la familia Rosaceae, la identificación de la S-RNasa como la determinante femenina, se hizo en mutantes naturales autocompatibles (AC) con mutaciones en el gen $S$-RNasa que inactivaban su producto (Yamame y Tao, 2009).

$\mathrm{Al}$ respecto, se ha propuesto que las S-RNasas actúan como agentes citotóxicos $S$-específicos (McClure et al., 1989). Estas ribonucleasas se incorporan a los TP durante su crecimiento por la matriz extracelular del tejido de transmisión del estilo, independientemente de si éstos son compatibles o incompatibles (Goldraij et al., 2006). Un dato importante que apoya que las S-RNasas se comportan como citotoxinas fue brindado por Huang et al. (1994), quienes por mutagénesis dirigida reemplazaron una de las dos histidinas conservadas en el sitio activo de la $\mathrm{S}_{3}$-RNasa de Petunia inflata; el resultado fue que los pistilos de las plantas transgénicas fueron incapaces de rechazar el polen $S_{3}$ debido a la incapacidad de la S3-RNasa para degradar el ARN del TP. 
SFB/SLF es la determinante masculina. El producto del alelo $S$ del polen se identificó recientemente en Petunia inflata (Sijacic et al., 2004). Este gen se conoce como $S L F$ (S-locus F-box gene) en solanáceas o $S F B$ ( $S$ hapolotype-specific F-box protein) en Rosaceae, y es miembro de una familia de proteínas que contiene una caja $\mathrm{F}$ hacia su extremo amino terminal. En general, esta proteína presenta dos dominios variables ( $\mathrm{Va}$ y $\mathrm{Vb}$ ) y dos dominios hipervariables ( $\mathrm{HVa}$ y $\mathrm{HVb}$ ) hacia el extremo carboxilo terminal (Ikeda et al., 2004; Yamame y Tao, 2009). De aquí en adelante la determinante masculina será referida como $S L F$.

Los miembros de la familia de proteínas con caja $\mathrm{F}$ se asocian con la vía de degradación de proteínas por el proteosoma 26S (Sullivan et al., 2003). La expresión de $S L F$ es haplotipo $S$-específica, y se restringe al polen (Sijacic et al., 2004). Para demostrar que SLF es la determinante masculina, Sijacic et al. (2004) ofrecieron una prueba contundente basada en el modelo de la interacción competitiva (Golz et al., 2001), el cual predice que el polen heteroalélico es aceptado por un estilo incompatible. En esta investigación se transformaron plantas incompatibles de $P$. inflata $S_{1} S_{1}$, $S_{1} S_{2}$, y $S_{2} S_{3}$, con el transgen $S L F_{-2}$ fusionado a un promotor específico de polen. Las plantas transgénicas resultantes produjeron polen homoalélico $\left(S L F_{2}+S_{2}\right)$ o heteroalélico $\left(S L F_{2}+S_{1}\right.$ o, $\left.S L F_{2}+S_{3}\right)$. Cuando las plantas transgénicas de $P$. inflata $S_{1} S_{1}+S L F_{-2}$ o $S_{2} S_{3}+$ $S L F-2$ fueron polinizadas con polen homoalélico o heteroalélico, sólo aceptaron el polen heteroalélico; esto demostró que hubo interacción competitiva (Figura 2) y que por, tanto $S L F$ es la determinante masculina.

La proteína SLF podría funcionar como una enzima ligasa tipo E3 (Sijacic et al., 2004). La degradación de proteínas vía el proteosoma $26 \mathrm{~S}$ involucra el marcaje por poliubiquitinación de las proteínas que se van a degradar. En este proceso las enzimas ligasas E3 representan un paso crucial en el control de la poliubiquitilación de la proteína blanco (target), ya que reconocen la señal de ubiquitilación en la proteína seleccionada y coordinan la transferencia de la ubiquitina (proteína de $76 \mathrm{kD}$ ) desde la ligasa E2 a un residuo de lisina de la proteína blanco (Sullivan et al., 2003). El reconocimiento de la proteína blanco y la transferencia de ubiquitina por las ligasas E3, puede ocurrir por la formación de un complejo proteico conocido como SCF-E3, el cual se conforma de cuatro proteínas: SKP1, culina, una proteína con caja F y RBX1 (Cardozo y Pagano 2004; Smalle y Vierstra, 2004).

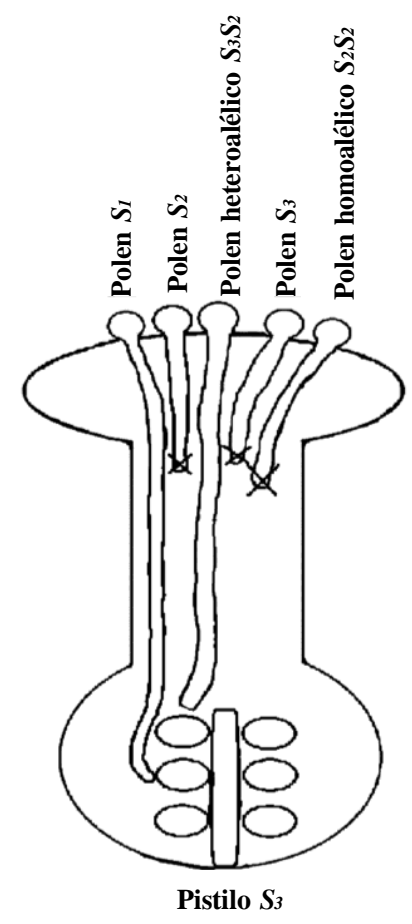

Figura 2. Interacción competitiva. Plantas heterócigas $S_{2} S_{3}$ que al ser polinizadas con polen homoalélico $S_{2} S_{2}$ inhiben el crecimiento de los tubos polínicos. Sin embargo, el polen heteroalélico $S_{2} S_{3}$ es capaz de crecer por el estilo hasta alcanzar el ovario, al igual que polen $S_{1}$.

\section{Factores no ligados al locus $S$ involucrados en el rechazo del polen}

A pesar de que las $S$-RNasas y $S L F$ determinan la especificidad de la incompatibilidad, evidencias genéticas indican que los productos de genes modificadores (GM) no ligados al locus $S$, son esenciales en la vía bioquímica del rechazo del polen (Ai et al., 1991; Murfett et al., 1996; Tsukamoto et al., 1999).

Los GM se clasifican en tres grupos (McClure et al., 2000). El Grupo I incluye genes cuyos productos regulan la expresión de las determinantes de la especificidad ( $S L F$ y $S$-RNasa). Un ejemplo de este grupo de genes lo describieron Tsukamoto et al. (1999; 2003) en una población silvestre de Petunia axillaries que segrega plantas autocompatibles y autoincompatibles; estos autores encontraron que la expresión de la $S_{13-R N a s a}$ está suprimida en las plantas AC por una mutación en el locus $M D F$.

El Grupo II comprende GM que regulan la actividad biológica de las determinantes de especificidad y, por tanto, su función se restringe a la incompatibilidad. De este grupo sólo dos GM han sido identificados con función confirmada en el rechazo del polen. Estos GM 
codifican a las proteínas HT-B y $120 \mathrm{~K}$ (McClure et al., 1999; Hancock et al., 2005).

En el Grupo III se encuentran los genes que funcionan en el rechazo del polen y en otros procesos de la interacción polen-pistilo. En este grupo podrían estar TTS, PELP III, Nap11, NaTrxh, NaStEP y SBP (O’Brien et al., 2004; Juárez-Díaz et al., 2006; Busot et al., 2008).

Por obvias razones, los GM más estudiados son $H T-B$ y $120 K$. Sus productos génicos ingresan a los TP cuando crecen por el estilo en $N$. alata (Goldraij et al., 2006). HT-B es una proteína de $8.6 \mathrm{kD}$, con un dominio constituido por 20 residuos de asparagina y ácido aspártico (dominio ND) hacia el extremo carboxilo (Kondo y McClure, 2008). El dominio ND esta flanqueado por dos motivos de cisteínas, CAACKC y CQTVCC en N. alata (Kondo y McClure, 2008). HT-B se expresa sólo en estilos maduros de plantas AI (McClure et al., 1999). El gen $H T-B$ está presente en tres géneros de la familia Solanaceae, Nicotiana, Petunia y Solanum (McClure et al., 1999; Kondo et al., 2002a, b; O'Brien et al., 2002; Sassa y Hirano, 2006). Su papel en la AI se confirmó mediante experimentos de pérdida de función en plantas transgénicas, las cuales perdieron la capacidad de rechazar su propio polen cuando $H T-B$ fue silenciado (McClure et al., 1999; O' Brien et al., 2002; Puerta et al., 2009).

La proteína $120 \mathrm{~K}$ es una arabinogalactoproteína con un motivo de extensina, que se localiza en las células del tejido de transmisión del estilo y es secretada a la matriz extracelular de este tejido tanto de especies autocompatibles como en autoincompatibles de Nicotiana (Hancock et al., 2005). El silenciamiento del gen $120 \mathrm{~K}$ por RNA interferente (RNAi) en híbridos de $N$. plumbaginifolia $\mathrm{x} N$. alata, abate la capacidad de reconocimiento del polen con el mismo haplotipo $S$ que el pistilo en $N$. alata (Hancock et al., 2005). En esta especie, $120 \mathrm{~K}$ forma complejos con las S-RNasas (CruzGarcía et al., 2005) e ingresa a los TP independientemente de su haplotipo $S$ (Lind et al., 1996; Goldraij et al., 2006). En cruzas compatibles y en etapas tempranas de la polinización de cruzas incompatibles, $120 \mathrm{~K}$ delimita el interior de las vacuolas del TP donde se encuentran contenidas las S-RNasas. No obstante, en etapas tardías (36 h) de una polinización incompatible, $120 \mathrm{~K}$ ya no se detecta en los TP (Goldraij et al., 2006). Estas evidencias sugieren que $120 \mathrm{~K}$ podría estar asociada con los sistemas membranosos en el TP y que su participación en el rechazo del polen sería en la liberación de las S-RNasas de la vacuola al citoplasma del TP, en coordinación con HT-B. Dado que en las plantas transgénicas con el gen $120 \mathrm{~K}$ silenciado no se afecta el transporte de las S-RNasas al TP, se ha sugerido que esta proteína funciona hasta que ha ingresado al TP (Hancock et al., 2005).

\section{Modelos para el rechazo del polen en sistemas de incompatibilidad basados en S-RNasa}

En la actualidad existen dos modelos que tratan de explicar cómo las S-RNasas pueden llevar acabo la inhibición del TP en cruzas incompatibles. Uno de ellos involucra la degradación de estas ribonucleasas (Hua et al., 2008) y el segundo su compartamentalización en vacuolas del TP (Goldraij et al., 2006; McClure, 2006).

Modelo de la degradación de las S-RNasas. Este modelo se basa fundamentalmente en la potencialidad que tiene SLF por su caja $F$, para participar en la vía de degradación de proteínas vía el proteosoma 26S. Muchas proteínas con caja $\mathrm{F}$ se caracterizan por ser componentes del complejo E3 de ligasa de ubiquitina, llamado SCF (Skp1, Cullin-F box), constituido por Skp1, cullin-1 una proteína con caja f y Rbx1. El complejo SCF está involucrado en la ubiquitinación específica y en la degradación de proteínas blanco mediante el proteosoma 26S, de forma que SLF podría ser parte de un complejo SCF (Moon et al., 2004).

Con base en lo anterior, Hua y Kao (2008) propusieron el modelo de la degradación de la S-RNasas. Para describir este modelo supondremos que un estilo $S_{1} S_{2}$ es polinizado con polen $S_{1}$, ejemplo en el que tanto la $S_{1}$ como la $\mathrm{S}_{2}$-RNasa producidas por el estilo son transportadas al interior del TP. En este caso la determinante masculina en el TP es $\mathrm{SLF}_{1}$, la cual mediará específicamente la ubiquitinación y la degradación de la $\mathrm{S}_{2}$-RNasa por el proteosoma $26 \mathrm{~S}$, pero no de la $\mathrm{S}_{1-}$ RNasa, por lo que esta ribonucleasa ejercerá su efecto citotóxico e inhibirá el crecimiento del TP $S_{1}$. En este modelo se sugiere que la interacción $S$-no específica entre SLF y la S-RNasa es más fuerte que la interacción SLF con la S-RNasa $S$-específica. Lo anterior se cumple muy bien en una cruza compatible; por ejemplo, si la polinización ocurre entre un estilo $S_{1} S_{2}$ y polen $S_{3}$, en el interior del TP se formarán complejos estables $\mathrm{S}_{1}$-RNasa$\mathrm{SLF}_{3}$ y $\mathrm{S}_{2}-\mathrm{RNasa}_{-} \mathrm{SLF}_{3}$, que provocarían la degradación de ambas S-RNasas y la estabilidad del ARN del TP no se verá afectada, de modo que TP alcanzaría el ovario (Figura 3). 


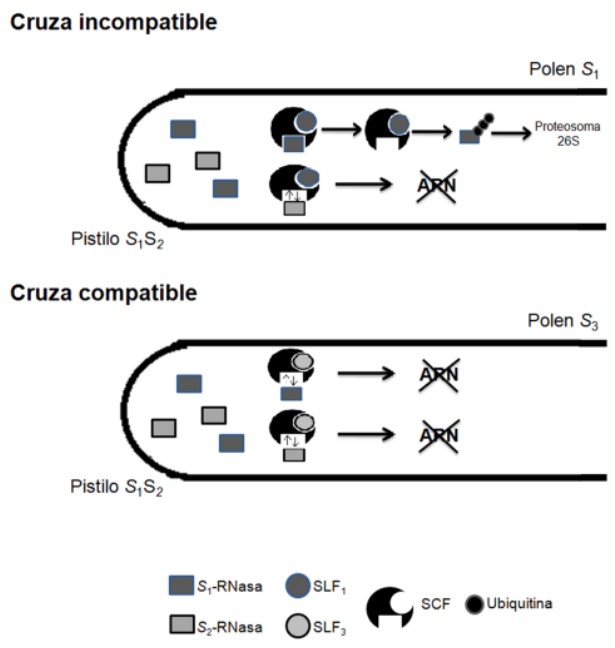

Figura 3. Modelo de la degradación de las $S$-RNasas. SLF forma parte del complejo SCF encargado de poliubiquitinar proteínas destinas a su degradación vía el proteosoma 26S. SLF posee un sitio de reconocimiento para todas las $S$-RNasas y un sitio alelo específico. En una cruza incompatible en un tubo polínico $S_{1}$ la interacción entre $S_{L F}$ y la $S_{2}$-RNasa conlleva a la degradación de la $S_{2}$ RNasa. Sin embargo, la interacción alelo específica entre $S_{L F}$ y la $S_{1}$-RNasa no es estable, por lo que la $S_{2}$-RNasa queda libre en el citoplasma para promover la degradación del ARN. En una cruza compatible la interacción no específica entre $\mathrm{SLF}_{3}$ y las $S_{1}$-RNasas y $S_{2}$-RNasas ocasiona la degradación de las dos $S$-RNasas, lo que permite el crecimiento del tubo polínico.

\section{Modelo de la compartamentalización de las S-RNasas}

Un modelo alternativo para explicar el rechazo del polen en sistemas basados en S-RNasa fue el propuesto por Goldraij et al. (2006), el cual sugiere que las SRNasas ingresan a los TP por endocitosis sin importar su haplotipo $S$, y se almacenan en una vacuola. Este modelo, además de la S-RNasa y SLF, incluye a los factores estilares $120 \mathrm{~K}$ y HT-B como componentes de un sistema selectivo del rechazo del polen secretado a la matriz extracelular del estilo. En esta situación, en una cruza compatible en donde un TP $S_{3}$ crece en un pistilo heterocigoto $S_{1} S_{2}$, el rechazo se evita debido a que tanto las $\mathrm{S}_{1}$-RNasas como la $\mathrm{S}_{2}$-RNasa permanecen secuestradas en la vacuola del TP, como consecuencia de la degradación de HT-B en el TP.

Por otro lado, en el caso de un polen $S_{1}$ o $S_{2}$ cuyos TP se desarrollen en un estilo $S_{1} S_{2}$, las vacuolas que contienen a las S-RNasas en el TP se romperán entre las 16 y $36 \mathrm{~h}$ de polinización, como respuesta a la interacción haplotipo $S$-específica entre SLF y la S-RNasa. La proteína HT-B, que ha ingresado al TP también y que probablemente se aloja en la misma vacuola donde están almacenadas las S-RNasas, permanecerá estable y mediará directa o indirectamente en que la vacuola se degrade y libere a las ribonucleasas al citoplasma del TP (Figura 4), en donde ejercerán su efecto citotóxico e inhibirá el crecimiento del TP (Goldraij et al., 2006).

\section{PROPUESTA DE UN MODELO MIXTO}

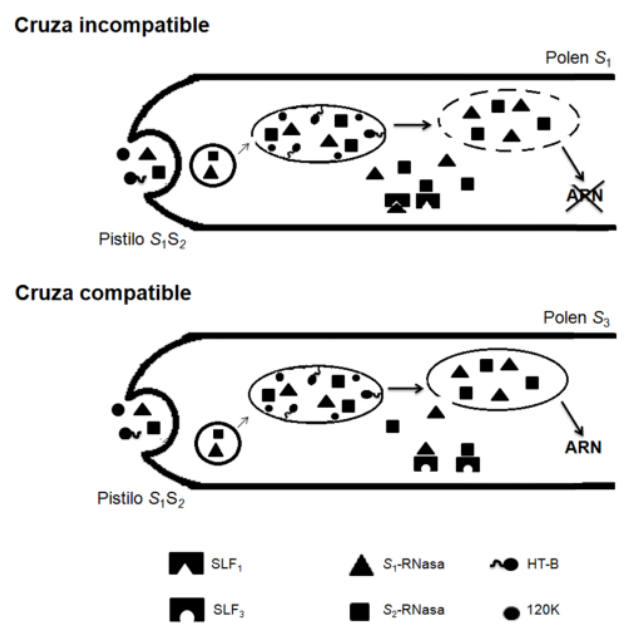

Figura 4. Modelo de la compartamentalización de las $S$ RNasas. Las $S$-RNasas ingresan al tubo polínico por endocitosis sin importar su haplotipo $S$. En una cruza incompatible con un pistilo $S_{1} S_{2}$ las $S$-RNasas son almacenadas en una vacuola junto con $120 \mathrm{~K}$ y HT-B. La interacción alelo específica entre $\mathrm{SLF}_{1}$ o $\mathrm{SLF}_{2}$ y las $S_{1-}$ RNasas y $S_{2}$-RNasas, conllevan a la estabilización de HT-B y al rompimiento de la vacuola del tubo polínico, $y$ dejan libres a las $S$-RNasas en su citosol para que lleven a cabo su efecto citotóxico. En una cruza compatible la interacción no específica entre SLF3, $S_{1}$-RNasas y $S_{2}$-RNasas, produce la degradación de HT-B y la estabilización de la vacuola, por lo que las $S$-RNasas permanecen almacenadas en la vacuola y el tubo polínico puede crecer hasta alcanzar el ovario.

Los dos modelos anteriores reflejan en gran medida los logros alcanzados en el entendimiento del mecanismo molecular del rechazo del polen en sistemas basados en SRNasa. Sin embargo, la información molecular vertida en ambos casos deja muchas incógnitas por resolver, lo que es resultado del desconocimiento de otros genes fuera del locus $S$, tanto del pistilo como del polen que completan la vía.

Otro aspecto que se deriva de los modelos es la aparente controversia que existe entre ambos, ya que mientras el modelo de la degradación de las S-RNasas establece que SLF es una proteína esencial que protege al tubo polínico de la acción citotóxica de las S-RNasas, y como no se han podido recuperar mutantes nulas para 
este gen en algunas solanáceas (Golz et al., 2001; McClure, 2009), el modelo de la compartamentalización propone que SLF causa el rechazo del polen, lo cual es consistente con las mutantes nulas para $S L F$ en Prunus (Sonneveld et al., 2005).

$\mathrm{Si}$ bien en apariencia estos modelos podrían ser irreconciliables, aquí se considera que podrían ser complementarios. Una mezcla de ambos modelos podría ser la siguiente: el proceso podría empezar con la formación de complejos entre SLF y la S-RNasa en el citoplasma del TP. En el ejemplo que se ilustra en la Figura 5, se presenta un pistilo homocigoto $S_{I} S_{I}$ que es polinizado con el polen $S_{1}$ (cruza incompatible) o $S_{2}$ (cruza compatible).

En la cruza incompatible (Figura 5A) se formarían complejos específicos $\mathrm{S}_{1}-\mathrm{RNasa}-\mathrm{SLF}_{1}$, que permitirían que la proteína HT-B permaneciera estable y activa en el TP para desencadenar la desestabilización y ruptura de la membrana de la vacuola que contiene las S-RNasas en el TP. Si esto ocurre, las S-RNasas se liberarían de forma masiva al citoplasma donde degradarían el ARN. Por otra parte, en una cruza compatible (Figura 5B) con polen $\mathrm{S}_{2}$ se formarían los complejos $S$-noespecíficos $S_{1}$-RNasa$\mathrm{SLF}_{2}$, los cuales serían re-ensamblados en el complejo $\mathrm{SCF}_{-} \mathrm{SLF}_{2}$ para permitir la poliubiquitinación de la S1-RNasa citoplasmática y que ésta sea degradada por el proteosoma 26S. Notablemente, este nuevo modelo propone también que la proteína HT-B podría ser un blanco del complejo SCF-SLF2, que la ubiquitinaría para ser degradada por el proteosoma 26S. Alternativamente, la proteína HT-B podría ser degradada por una proteasa de síntesis de novo en el tubo polínico, ya que su programa de síntesis de proteínas sigue activo. Cualquiera que sea la vía de degradación de HT-B, lo importante es que esta proteína no estaría disponible para mediar la ruptura de la vacuola con las S-RNasas en el tubo polínico, la cual permanecería intacta y bloquearía así la actividad citotóxica de las S-RNasas para permitir el crecimiento del TP al ovario.

En este modelo unificado la actividad de la S-RNasa que no se degrada por el proteosoma $26 \mathrm{~S}$ no sería suficiente para provocar la muerte de los tubos polínicos, y sólo funcionaría para darle especificidad a la respuesta de incompatibilidad.

Sin embargo, para disponer de un modelo que describa con más detalle el mecanismo molecular del rechazo del polen en sistemas basados en S-RNasas, la investigación futura deberá estar dirigida a responder algunas preguntas, como: ¿Cuáles son las proteínas estilares y del polen que integran la vía bioquímica del rechazo del polen? ¿Cómo se degrada la proteína HT-B en el tubo polínico y si depende de la interacción $S$-específica entre
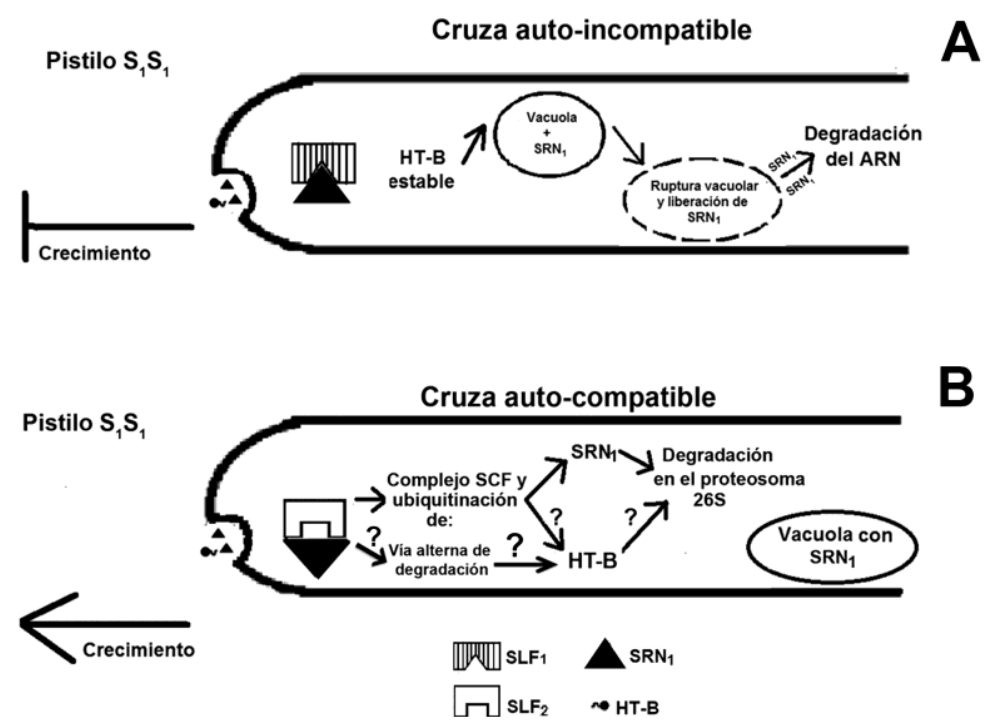

Figura 5. Modelo unificado del rechazo del polen en sistemas basados en S-RNasas. En este modelo se integran los mecanismos propuestos por Hua y Kao (2008; degradación de la S-RNasa) y por Goldraij et al. (2006; compartamentalización de la S-RNasa). Aquí se propone que la interacción específica permitiría que la proteína HT-B permaneciera estable y activa en el TP de una cruza incompatible (A), para desencadenar la ruptura de la vacuola que contiene a las S-RNasas (SRN), y que su liberación masiva inhibiera el crecimiento del TP. En una cruza compatible (B) el escenario sería diferente, ya que la interacción específica propiciaría el ensamblaje de un complejo SCF-SLF que poliubiquitinara a la RNasa y probablemente a HT-B, para ser degradadas por el proteosoma 26S. Alternativamente, HT-B podría ser degradada por una proteasa de síntesis de novo. En cualquier caso, la degradación de HT-B inhibiría la ruptura de la vacuola con las RNasas, lo que evitaría su actividad citotóxica y permitiría el crecimiento del TP. 
SLF y la S-RNasa? ¿Cuáles son las señales que promueven la liberación de las S-RNasas de las vacuolas de TP en cruzas incompatibles? y ¿Cuál es el mecanismo molecular mediante el cual HT-B propicia la desestabilización de la vacuola que contiene a las SRNasas en el TP en cruzas incompatibles?

\section{BIBLIOGRAFÍA}

Ai Y, E Kron, T -h Kao (1991) $S$-alleles are retained and expressed in a self-compatible cultivar of Petunia hybrida. Mol. Gen. Genet. 230:353-358.

Anderson M, A E C Cornish, S L Mau, E G Williams, R Hoggart, A Atkinson, IBönig, B Grego, R Simpson, P J Roche, J D Haley, J D Penschow, H D Niall, G W Tregear, J P Coughlan, R J Crawford, A E Clarke (1986) Cloning of cDNA for a stylar glycoprotein associated with expression of self-incompatibility in Nicotiana alata. Nature 321:38-44.

Busot G, B McClure, C Ibarra-Sánchez, K Jiménez-Durán, S Vázquez-Santana, F Cruz-García (2008) Pollination in Nicotiana alata stimulates synthesis and transfer to the stigmatic surface of NaStEP, a vacuolar Kunitz proteinase inhibitor homologue. J. Exp. Bot. 59:3187-3201.

Cardozo T, M Pagano (2004) The SCF ubiquitin ligase: insights into a molecular machine. Nature Rev. Mol. Cell Biol. 5:739-751.

Charlesworth D, X Vekemans, V Castric, S Glémin (2005) Plant self-incompatibility systems: a molecular evolutionary perspective. New Phytol. 168:61-69.

Cornish E C, M A Anderson, A E Clarke (1988) Molecular aspects of fertilization in flowering plants. Annu. Rev. Cell Develop. Biol. 4:209-228.

Cruz-García F, B McClure (2001) Sexual incompatibility. In: Current Trends in the Embryology of Angiosperms. S S Bhojwani, WY Soh (eds). Kluwer Academic Publishers. Netherlands. pp:167196.

Cruz-García F, C N Hancock, D Kim, B McClure (2005) Stylar glycoproteins bind to $S$-RNase in vitro. The Plant J. 42:295304.

de Nettancourt (1977) Incompatibility in angiosperms. Sexual Plant Reprod. 10:185-199.

de Nettancourt (2001) Incompatibility in Angiosperms: Monographs on Theoretical and Applied Genetics 3. Springer-Verlag. New York. $312 \mathrm{p}$.

Dickinson H G, M J C Crabbe, T Gaude (1992) Sporophytic selfincompatibility systems: $S$ gene products. Internatl. Rev. Cytol. 140:525-561.

Entani T, M Iwano, H Shiba, S Takayama, K Fukui, A Isogai (1999) Centromeric localization of an $S$-RNase gene in Petunia hybrida. Theor. Appl. Genet. 99:391-397.

Goldraij A, K Kondo, C B Lee, C N Hancock, M Sivaguru, S Vazquez-Santana, S Kim, T E Phillips, F Cruz-García, B McClure (2006) Compartmentalization of $S$-RNase and HT-B degradation in self-incompatible Nicotiana. Nature 439:805810 .

Golz J F, H Y Oh, V Su, M Kusaba, E Newbigin (2001) Genetic analysis of Nicotiana pollen-part mutants is consistent with the presence o fan $S$-ribonuclease inhibitor at the $S$ locus. Proc. Nat. Acad. Sci. 98:15372-15376.

Hancock C N, L Kent, B McClure (2005) The stylar $120 \mathrm{kDa}$ glycoprotein is required for $S$-specific pollen rejection in Nicotiana. The Plant J. 43:716-723.

Hua Z, T-h Kao (2008) Identification of major lysin residues of $S_{3-}$ RNase of Petunia inflata involved in ubiquitin-26S proteosomemediated degradation in vitro. The Plant J. 54:1094-1104.
Hua Z H, A Fields, T-h Kao (2008) Biochemical models for $S$-RNase based self-incompatibility. Mol. Plant 1:575-585.

Huang S, H-S Lee, B Karunanandaa, T-h Kao (1994) Ribonuclease activity of Petunia inflata $S$ protein is essential for rejection of self-pollen. The Plant Cell 6:1021-1028.

Ikeda K, I Boris, K Ushijima, H Yamame, N R Haunck, R Nakano, H Sassa, A F Iezzoni, J R Kohn, $R$ Tao (2004) Primary structural features of the $S$-haplotype-specific $\mathrm{F}$ box protein, SFB, in Prunus. Sexual Plant Reprod. 16:235-243.

Juárez-Díaz J, B McClure, S Vázquez-Santana, A Guevara-García, P León-Mejía, J Márquez-Guzmán, F Cruz-García (2006) A novel Thioredoxin $h$ is secreted in Nicotiana alata and reduces S-RNase in vitro. J. Biol. Chem. 281:3418-3424.

Kao T-h, A G McCubbin (1996) How flowering plants discriminate between self and non-self pollen to prevent inbreeding. Proc. Nat. Acad. Sci. 93:12059-12065.

Kondo K, B McClure (2008) New microsome-associated HT-family proteins from Nicotiana respond to pollination and define an HT/NOD-24 protein family. Mol. Plant 8:634-644.

Kondo K, M Yamamoto, $R$ Itahashi, T Sato, H Egashira, T Hattori, Y Kowyama (2002a) Insights into the evolution of self-comapatibility in Lycopersicum from a study of stylar factors. The Plant J. 30:142-153.

Kondo K, M Yamamoto, D Matton, T Sato, M Hirai, S Norioka, T Hattori, Y Kowyama (2002b) Cultivated tomato has defects in both $S$-RNase and $H T$ genes required for stylar function of selfincompatibility. The Plant J. 29:627-636.

Lind J L, I Bönig, A E Clarke, M A Anderson (1996) A stylespecific $120 \mathrm{kDa}$ glycoprotein enters pollen tubes of Nicotiana alata in vivo. Sexual Plant Reprod. 9:75-86.

McClure B (2006) New views of $S$-RNase-based self-incompatibility. Curr. Op. Plant Biol. 9:639-646.

McClure B (2009) Darwin's foundation for investigating selfincompatibility and the progress toward a physiological model for $S$-RNase-based SI. J. Exp. Bot. 60:1069-1081.

McClure B, V Haring, P R Ebert, M A Anderson, R J Simpson, F Sakiyama, A E Clarke (1989) Style self-incompatibility gene products of Nicotiana alata are ribonucleases. Nature 342:955957.

McClure B, B Mou, S Canevascini, R Bernatzky (1999) A small asparagine-rich protein required for $S$-allele-specific pollen rejection in Nicotiana. Proc. Nat. Acad. Sci. 96:13548-13553.

McClure B, F Cruz-García, B Beecher, W Sulaman (2000) Factors affecting inter and intra specific pollen rejection in Nicotiana. Ann. Bot. Suppl. 85A:113-123.

McClure B, V Frankling-Tong (2006) Gametophytic selfincompatibility: understanding the cellular mechanisms involved in self pollen tube inhibition. Planta 224:233-245.

Moon J, G Parry, M Estelle (2004) The ubiquitin-proteasome pathway and plant development The Plant Cell 16:3181-3195.

Murffet J, T L Atherto, B Mou, C S Gasser, B McClure (1994) $S$ RNase expressed in transgenic Nicotiana causes $S$-allele specific pollen rejection. Nature 367:563-566.

Murffet J, T J Strabala, D M Zurek, B Mou, B Beecher, B McClure (1996) $S$-RNase and inter-specific pollen rejection in the genus Nicotiana: multiple pollen- rejection pathways contribute to unilateral incompatibility between selfincompatible and self-compatible species. The Plant Cell 8:943958.

Newbigin E, A M Anderson, A Clarke (1993) Gametophytic selfincompatibility systems. The Plant Cell 5:1315-1324.

Newbigin E, M K Uyenoyama (2005) The evolutionary dynamics of self-incompatibility systems. Trends Genet. 21:500-505.

O'Brien M, C Kapfer, G Mayor, M Laurin, C Bertrand, K Kondo, Y Kowyama, D P Matton (2002) Molecular analysis of the stylar-expressed Solanum chacoense small asparagine-rich 
protein family related to the HT modifier of gametophytic selfincompatibility in Nicotiana. The Plant J. 32:985-996.

O'Brien M, G Major, S Chanta, D Matton (2004) Isolation of a $S$ RNase protein from Solanum chacoense: identification of an SBP1 (Ring finger protein) ortholog. Sexual Plant Reprod. 17:81-87.

Puerta A R, K Ushijima, T Koba, H Sassa (2009) Identification and functional analysis of pistil self-incompatibility factor $H T-B$ of Petunia. J. Exp. Bot. 60:1309-1318.

Sassa H, T Nishio, Y Kowyama, H Hirano, T Koba, H Ikehashi (1996) Self-incompatibility $(S)$ alleles of the Rosaceae encode members of a distinct class of the $\mathrm{T} 2 / \mathrm{S}$ ribonuclease super family. Mol. Gen. Genet. 27:222.

Sassa H, H Hirano (2006) Identification of a new class of pistil-specific proteins of Petunia inflata that is structurally similar to, but functionally distinct from the self-incompatibility factor HT. Mol. Genet. Genom. 275:97-104.

Sijacic P, X Wang, A L Skirpa, Y Wang, P E Dowd, A G McCubbin, S Huang, T-h Kao (2004) Identification of the pollen determinant of $S$-RNase-mediated self-incompatibility. Nature 429:302-305.

Smalle J, R D Vierstra (2004) The ubiquitin 26S proteasome proteolytic pathway. Annu. Rev. Plant Biol. 55:555-590.

Sonneveld T, K R Tobutt, S P Vaughan, T P Robbins (2005) Loss of pollen-S function in two self-compatible selections of Prunus avium is associated with deletion/mutation of an $S$-haplotype Fbox gene. The Plan Cell 17:37-51.

Sullivan J A, K Shirasu, X W Deng (2003) The diverse roles of ubiquitin and the $26 \mathrm{~S}$ proteasome in the life of plants. Nature Rev. Genet. 4:948-958.

Tanksley S D, F Loaiza-Figueroa (1985) Gametophytic selfincompatibility is controlled by a single major locus on chromosome 1 in Lycopersicon peruvianum. Proc. Nat. Acad. of Sci. 82:5093-5096.

Tsukamoto T, T Ando, $\mathbf{H}$ Kokubun, $\mathrm{H}$ Watanable, M Masada, $\mathrm{X}$ Zhu, E Marchesi, T-h Kao (1999) Breakdown of selfincompatibility in a natural population of Petunia axillaris (Solanaceae) in Uruguay containing both self-incompatible and self-compatible plants. Sexual Plant Reprod. 12:1432-2145.

Tsukamoto T, T Ando, H Kokubun, H Watanable, T Sato, M Masada, E Marchesi, T-h Kao (2003) Breakdown of selfincompatibility in a natural population of Petunia axillaris caused by a modifier locus that suppresses the expression of an $S$-RNase gene. Sexual Plant Reprod. 15:255-263.

Xue Y B, R Carpenter, H G Dickinson, E S Coen (1996) Origin of allelic diversity in Antirrhinum $S$ locus RNase. The Plant Cell 8:805-814.

Yamame H, R Tao (2009) Molecular basis of self-(in)compatibility and current status of $S$-genotyping in Rosaceous fruit trees. J. Japan. Soc. Hort. Sci. 78:137-157. 